\title{
Initial experience of video assisted thoracoscopic pneumonectomy
}

\author{
Stewart R Craig, William S Walker
}

\begin{abstract}
Background - Preliminary experience of video assisted thoracoscopic pneumonectomy in six patients with bronchogenic carcinoma is described.

Methods - Four left and two right pneumonectomies were performed under video thoracoscopic imaging. Thoracoscopic instruments were passed through two separate stab incisions on the lateral chest wall and a separate $6 \mathrm{~cm}$ submammary incision was also created to allow further access for instrumentation and removal of the resected lung. In this initial experience resection was restricted to patients with bronchogenic carcinomas of less than $6 \mathrm{~cm}$ in diameter who had no involvement of the mediastinum.
\end{abstract}

Results - There were no operative deaths and no complications attributable to the technique. One patient developed postoperative atrial fibrillation and a small sacral sore and one patient was readmitted with abdominal pain and pyrexia which settled following exclusion of post pneumonectomy empyema. The remaining four patients made a rapid uncomplicated postoperative recovery with less pain and discomfort than that normally associated with a standard posterolateral thoracotomy. Postoperatively the mean (SD) patient controlled morphine consumption was $1.36(1.90) \mathrm{mg}$ per hour in the first 36 hours compared with the unit mean for open thoracotomy of $1.73(1.68) \mathrm{mg}$ per hour. The mean linear visual analogue pain score was $15 \cdot 4(15 \cdot 6)$ in the first 24 hours compared with the unit mean for open thoracotomy of $34 \cdot 5(8 \cdot 5)$.

Conclusions - Video assisted thoracoscopic pneumonectomy can be performed safely in patients who have stage $I$ and stage II bronchogenic carcinomas, up to $6 \mathrm{~cm}$ in diameter, with no mediastinal involvement on mediastinoscopy and thoracic computed tomographic assessment. This technique may result in less postoperative pain and discomfort and should allow a quicker return to normal activities. (Thorax 1995;50:392-395)

Keywords: thoracoscopic surgery, pneumonectomy.

Major pulmonary resection for bronchogenic carcinoma by video assisted thoracoscopic techniques has, until recently, been limited mainly to pulmonary lobectomy. Video assisted thoracoscopic pneumonectomy has been re- ported from a few centres with encouraging $\frac{\bar{\sigma}}{\bar{c}}$ early results. ${ }^{1-3}$ We have performed six video

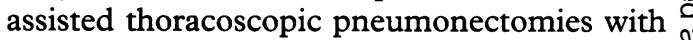
no complications or mortality attributable to 0 the technique.

\section{Methods}

PATIENTS

Sixty two patients referred to the thoracic surgical unit between April 1992 and May $1994 \mathrm{~N}$ underwent video assisted thoracoscopic lob- 을 ectomy or pneumonectomy. Of these $62 \rightarrow$ patients six had a video assisted thoracoscopic $\frac{7}{0}$ pneumonectomy performed for bronchogenic carcinoma. Fitness for surgery was based on $\vec{\bullet}$ standard criteria for open thoracotomy (ad- of equate spirometric values for pneumonectomy, satisfactory arterial blood gases, and stable cardiac status).

Preoperative chest radiography revealed two ฏ

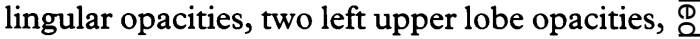
one right upper lobe opacity, and one middle $\overrightarrow{\vec{A}}$ lobe opacity. Thoracic computed tomography was performed in all patients and confirmed $כ$ the chest radiograph findings and either demonstrated no mediastinal lymph nodes or nodes of less than $5 \mathrm{~mm}$ in diameter. Fibreoptic bronchoscopy with biopsy diagnosed two upper lobe and one middle lobe bronchial carcinomas. In the other three cases no endobronchial abnormality was noted at bronchoscopy; however, in one case washings demonstrated cells compatible with origin from an adenocarcinoma from a lingular bronchus. Mediastinoscopy was performed in five of the six cases and excluded the presence of mediastinal lymph node in- o volvement. Patients were screened for distant $N$ metastatic spread by liver ultrasonography and N by isotopic bone scan (if indicated on clinical 0 or biochemical grounds). Each patient gave 0 specific consent for resection by video assisted $\overparen{\Phi}$ thoracoscopic technique; however, the possible $\stackrel{?}{?}$ need to revert to standard open posterolateral 0 thoracotomy was also explained to the patient.

Two patients with carcinomas located in the $\cong$ lingula required a pneumonectomy because of $\stackrel{\mathbb{Q}}{\varrho}$ involvement of the adjacent lower lobe at the time of initial video assessment. Three patients 8 with upper lobe carcinomas required pneumonectomy because of tumour at the origin of the upper lobe bronchus in two and carcinomain-situ within the left main bronchus in the other. The patient with a middle lobe squamous carcinoma required a pneumonectomy as the tumour crossed the oblique fissure to involve the lower lobe over a large area and extended along the middle lobar vein to the pericardium. 
Clinical details of the six patients

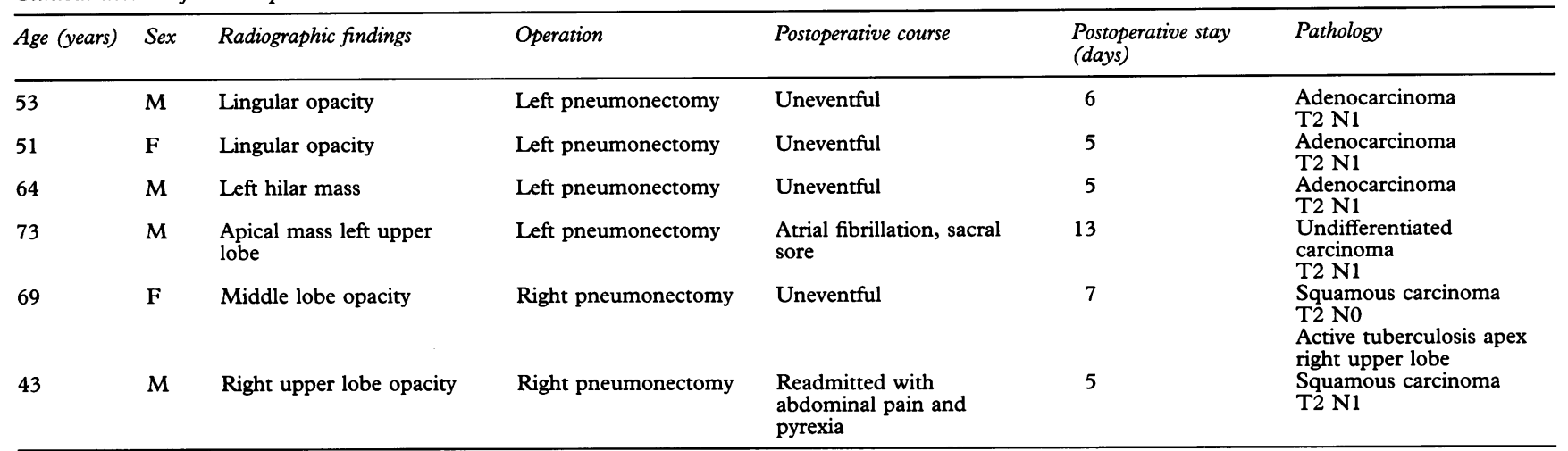

SURGICAL TECHNIQUE

Video assisted thoracoscopic pneumonectomy was performed with the patient prepared, anaesthetised, and positioned as for a standard posterolateral thoracotomy. Instruments for thoracotomy were immediately available along with a selection of vascular clamps. Single lung anaesthesia was achieved with a double lumen endobronchial tube and intraoperative monitoring included continuous radial arterial pressure, electrocardiogram, oxygen saturation, and end tidal carbon dioxide pressure. Access in each case was gained by two or three stab incisions on the lateral chest wall which allowed insertion of the videothoracoscopic system and a selection of thoracoscopic dissecting and stapling instruments. ${ }^{3}$ In addition, a $6 \mathrm{~cm}$ submammary incision was created to allow removal of the resected lung and to permit additional access for instrumentation.

Once the decision to proceed to pneumonectomy was made the pulmonary ligament was divided with a diathermy hook and the lower pulmonary vein identified. The inferior pulmonary vein was divided using an EndoGIA 30 V3 vascular stapler (Auto Suture Co, Ascot, UK). The bronchus was then cleared of adjacent lymphatics and the bronchial arteries secured with Endo-Clips (Auto Suture Co). In the first four patients the bronchus was then stapled with a TA30 Roticulated bronchial stapler (Auto Suture Co) passed through the submammary incision, and the bronchus was divided distal to this. In the other two patients an Endo-GIA 604.8 stapler was used. The superior pulmonary vein was then divided and the pulmonary artery separated from surrounding hilar tissue. The pulmonary artery was controlled proximally with a vascular clamp passed through the submammary incision and divided with the Endo-GIA 30 V3 vascular stapler. The closure line on the pulmonary artery was carefully inspected before slowly releasing the vascular clamp. The resected lung was placed in a polythene bag and withdrawn from the chest through the submammary incision without the need for rib retraction. The bronchial stump was tested under water and confirmed to be airtight. All central hilar nodes and any clinically suspicious mediastinal nodes were excised. No difficulty was encountered in clearing subcarinal glands. The pleural cavity and wounds were lavaged with sterile water and Kefadol (Dista Products Ltd, Basingstoke, UK) was instilled. A single basal chest drain was inserted and the wounds closed in layers with Vicryl sutures (Ethicon Ltd, Edinburgh, UK).

The patients were admitted to a high dependency unit (HDU) for the first 48 hours postoperatively and subsequently transferred to the general thoracic ward until discharge. During their stay in the HDU the visual analogue pain score $(0=$ pain free, $100=$ worst pain imaginable) was measured every four hours, together with morphine consumption which was delivered by a patient controlled analgesia device.

\section{Results}

There were four men and two women with a mean age of 59 (11) years. The mean operating time was 233 (71) minutes (range 140-330 minutes) and the mean blood loss was 294 (121) $\mathrm{ml}$ (range $80-440 \mathrm{ml}$ ). The intercostal drain was removed after 24 hours in each case. The mean patient controlled morphine consumption was $1.36(1.90) \mathrm{mg}$ per hour during the first 36 hours postoperatively. This compares favourably with the thoracic surgical unit mean hourly consumption of morphine of 1.73 $(1.68) \mathrm{mg}$ per hour for open thoracotomy. The mean linear visual analogue pain score was $15 \cdot 4(15 \cdot 6)$ for the first 24 hours compared with $34.5(8.5)$ for open thoracotomy. No patients required postoperative intercostal nerve blocks. Five of the six patients made an uncomplicated postoperative recovery and were discharged at a mean of six days. The sixth patient developed atrial fibrillation, which responded rapidly to intravenous digoxin, and a small sacral sore. Following these minor complications he made a good postoperative recovery and was discharged well on his 13th postoperative day.

One patient was readmitted from the original referring hospital one week following discharge with suspected post pneumonectomy empyema. He had developed right sided abdominal pain and pyrexia, which necessitated admission, two days before transfer. The referring hospital reported that percutaneous as- 
piration of fluid from his pneumonectomy space had yielded a scanty growth of Staphylococcus aureus on culture for which he was treated with parenteral flucloxacillin and gentamicin. On transfer his abdominal pain persisted but an abdominal ultrasound scan, white blood count, and urinalysis were all normal. The pneumonectomy space was drained with an underwater seal drain in view of the alleged infection and three separate cultures of the clear straw coloured space fluid were reported sterile. The patient remained clinically well and his abdominal pain and pyrexia settled. The pneumonectomy space was irrigated for three days with sterile saline containing flucloxacillin and the drain was then removed without recurrence of his symptoms. The clinical details of each patient are listed in the table.

The mean postoperative follow up to date is 12 months (range 3-24 months). Four patients remain well with no clinical or radiological evidence of recurrence. Two patients, one with adenocarcinoma and one with undifferentiated carcinoma, developed distant metastatic disease which was diagnosed in each case seven months after surgery. Neither had evidence of local or regional recurrence on chest radiography or on thoracic computed technology. One developed a single cerebral metastasis which was treated by craniotomy. The other patient developed bony metastases which were treated with palliative radiotherapy. Both patients are currently alive and at home.

\section{Discussion}

Video assisted thoracoscopic pulmonary resection for bronchogenic carcinoma is a recent development. This technique was initially confined to wedge resection of small peripheral tumours ${ }^{45}$ which raised concerns, from an oncological viewpoint, about the completeness of resection. These fears appear to be confirmed by Ginsberg and Rubinstein ${ }^{6}$ and by Warren and $\mathrm{Faber}^{7}$ who have found a higher incidence of local/regional tumour recurrence in patients subjected to wedge or segmental resection than with lobectomy. This increased risk of local recurrence after limited resection may be partly explained by the relatively high rate of lymphatic vessel invasion seen in peripheral bronchogenic carcinomas, even when there is no involvement of the regional lymph nodes. ${ }^{8}$ It therefore follows that video assisted pulmonary resection should not result in the abandonment of traditionally accepted practice in patients with bronchogenic carcinoma who undergo resection by formal thoracotomy.

Once video assisted thoracoscopic techniques were developed it became possible to perform a dissectional lobectomy that compared favourably with standard open thoracotomy. ${ }^{9-11}$ The new technique resulted in less postoperative pain and discomfort than open thoracotomy and allowed a quicker return to normal activities.

Although limited, our experience and that of others ${ }^{1-3}$ confirms that it is possible to perform a pneumonectomy using video assisted thora- coscopic techniques. This technique is suitable for selected patients with bronchogenic carcinoma who have stage I and II disease, with a normal mediastinum and clear access to the hilar structures. Patients who undergo video assisted pneumonectomy appear to have all the benefits of the minimal access procedure with the important additional benefit that they are not subject to postoperative air leaks as can occur in patients with incomplete fissures who undergo video assisted thoracoscopic lobectomy. Clinical postoperative progress is therefore even more rapid in patients who undergo video assisted thoracoscopic pneumonectomy.

It is mandatory to gain proximal control of the pulmonary artery with a vascular clamp and desirable to clamp the main pulmonary veins centrally before division with endoscopic stapling devices, as we have had two instances in cases undergoing lobectomy where the stapler has cut but failed to staple the major vessels. Both cases were resolved without incident but necessitated expeditious conversion to open thoracotomy.

We believe it is important to place the resected lung (or lobe) into a bag before removing it from the thoracic cavity in order to prevent tumour cell implantation. This phenomenon has been reported in surgical "port hole" wounds performed for thoracic ${ }^{12}$ and $a b$ dominal ${ }^{13}$ malignancy.

Giudicelli et $a l^{14}$ have also reported a variation of video assisted pneumonectomy using a mini thoracotomy. Their technique lies between conventional thoracotomy and video assisted thoracoscopic surgery as the mini thoracotomy is used both for direct vision of the operative field and to allow access for specially adapted surgical rather than thoracoscopic instruments. Direct vision of the operative field through the mini thoracotomy may not, however, afford the surgeon the excellent view of the hilar structures and apical region obtained using purely video imaged techniques. Although no major muscles are divided with this technique, significant rib separation is required which may negate the previously described benefits of a minimal access procedure. Preliminary experience with video assisted pulmonary resection (pneumonectomy and lobectomy) suggests that this technique offers many potential advantages to the patient when compared with standard open thoracotomy. There may be other significant potential benefits including reduced tissue trauma and postoperative immunosuppression with improved pulmonary function and mobility. We are currently attempting to quantify these advantages in a prospective randomised trial.

1 Roviaro G, Varoli F, Rebuffat C, Vergani C, D'Hoore A, Scalambra SM, et al. Major pulmonary resections: pneumonectomies and lobectomies. Ann Thorac Surg 1993;56: 779-83.

2 McKenna RJ Jr. Lobectomy by video-assisted thoracic surgery with mediastinal node sampling for lung cancer. $f$ Thorac Cardiovasc Surg 1994;107:879-82.

3 Walker WS, Carnochan FM, Mattar S. Video-assisted thoracoscopic pneumonectomy. Br $\mathcal{F}$ Surg 1994;81:81-2

Wakabayashi A Expanded applications of diagnostic. therapeutic thoracoscopy. $\mathcal{F}$ Thorac Cardiovasc Surg 1991; 102:721-3. 
5 Donnelly RJ, Page RD, Cowen ME. Endoscopy assisted microthoracotomy: initial experience. Thorax 1992;47: 490-3.

6 Ginsberg RJ, Rubinstein L. A randomized comparative trial of lobectomy vs segmentectomy for patients with T1 N0 non-SCLC lung cancer. Lung Cancer 1991; 7(Suppl):83A

7 Warren WH, Faber LP. Segmentectomy versus lobectomy in patients with stage I pulmonary carcinoma. $\mathcal{F}$ Thorac

8 Ichinose Y, Yano T, Yokoyama $\mathrm{H}$, Inoue $\mathrm{T}$, Asoh $\mathrm{H}$, Katsuda Y. The correlation between tumor size and lymphatic vessel invasion in resected peripheral stage I non-small cell lung cancer. $f$ Thorac Cardiovasc Surg 1994;108:684-6.

9 Kirby TJ, Mack MJ, Landreneau RJ, Rice TW. Initial experience with video-assisted thoracoscopic lobectomy. Ann Thorac Surg 1993;56:1248-53.

10 Walker WS, Carnochan FM, Pugh GC. Thoracoscopic pulmonary lobectomy. $\mathcal{F}$ Thorac Cardiovasc Surg 1993;106 1111-7.

11 Walker WS, Carnochan FM, Tin M. Thoracoscopy assisted pulmonary lobectomy. Thorax 1993;48:921-4.

12 Thurer R. Video-assisted thoracic surgery. Ann Thorac Surg 1993;56:199-200.

13 Nduka CC, Monson JRT, Menzies-Gow N, Darzi A. Abdominal wall metastases following laparoscopy. Br f Surg 1994;81:648-52.

14 Giudicelli R, Thomas P, Lonjon T, Ragni J, Bulgare JC Ottomani $\mathbf{R}$, et al. Major pulmonary resection by video assisted mini-thoracotomy. Eur f Cardiothorac Surg 1994; 8:254-8.

\section{The Crofton Cup II: non-tobacco sponsored golf goes full strength - players please}

"I'm looking for the organiser of the golf - it's Ainslie Moore here - I can play you know."

It is the evening before the competition. Tee times confirmed, lists made, dinner for 50 booked, weather prayed for and here on cue is the Reverend Moore.

"Of course. Delighted. See you at the clubhouse."

Dawn breaks, skies not quite as blue as last year and a quality links breeze is lifting the slates. Arran is sitting pretty off to the west and the sea is stunning. Would the golf be difficult? Try playing in a filmset - easier perhaps if Teuton than Celt.

The morning round is played by a favoured few, joined by some locals who had heard of the event and wanted to play in the sweep promptly donated by the winner to the charity.

After lunch the tee is busy with chest physicians, GPs, and a motley bunch from commerce - business people, bankers and accountants. Our worry that this Open qualifier course would be a mite tricky proved marginally correct. The motley bunch did very well though, and $\mathrm{Mr}$ Teuton from the bank won the trophy. Reverend Moore, as predicted, won the ecclesiastical medal, and the physician from Edinburgh choked on his pipe (rarely lit) when a colleague from the West tabled his score of 158 .

"Quite remarkable," as we (including $\mathrm{Dr}$ 158) drowned in ungracious tears of laughter.

That the event was competitive in spirit was acceptable - but we did not expect sequential birdies from the mild mannered GP or the dead heat in the longest drive which, according to the Barassie (now European Club Cham- pions) Secretary, was probably unique in the history of golf - an assessment quite agreeable to the successful young fellow and Dr Pipe from Edinburgh (mutual nodding). Another Dr Pipe (only-lit-when-fishing) took the prize for the par threes and is negotiating his prize with our friendly clubmaker (Macpherson of Troon).

So ended another Crofton Cup. Since we wish to develop our concerns about tobacco sponsorship in golf beyond the Crofton, the Physiks Plate is our UK wide response. Details of this event - for a prize determined by submission of the highest Stableford score under medal conditions at the competitor's own course during June - will be sent out after application and registration (fee $f^{5}$ ) at the Scottish Office of the British Lung Foundation, Royal College of Physicians and Surgeons of Glasgow, 234-242 St Vincent Street, Glasgow G2 5RJ. This will be an open competition, and we seek support from all who are impressed by an obligation to act in favour of tobacco sponsorship-free sport. Although we in the West of Scotland hold the world record for lung cancer, mainly due to the efforts of tobacco, we do so reluctantly. In terms of sporting records at the highest level, distribution is more consistently international and, surprisingly, occurs in the complete absence of tobacco.

On the basis of obtuse rationality, perhaps readers elsewhere in the world might also wish to take part. Players please.

KENNETH ANDERSON 